\title{
A RESTAURAÇÃO FLUVIAL E A BUSCA DE RECONCILIAÇÃO DA EUROPA COM OS SEUS CURSOS D'ÁGUA: O QUE O BRASIL DEVE APRENDER COM ESTA EXPERIÊNCIA?
}

\author{
MAURÍCIO MEURER ${ }^{1}$ \\ Universidade Federal de Pelotas
}

\section{Introdução}

A restauração fluvial é um assunto relativamente desconhecido no Brasil, mas nos países da União Europeia e em alguns países desenvolvidos esse vem sendo um dos temas prioritários na pauta de discussões das autoridades responsáveis pela gestão dos recursos hídricos.

A exploração dos recursos hídricos na Europa é muito antiga; ao longo dos séculos os cursos d'água foram utilizados para os mais diversos fins, como o abastecimento de água potável, a irrigação, a pesca, a movimentação das rodas d'água que transformavam a força hidráulica em força mecânica, a navegação e o transporte de mercadorias, entre outras atividades. Por conta dessa intensa exploração, os rios europeus foram sendo submetidos, ao longo de sua história, a incontáveis obras de controle e estabilização do canal (desvios, canalizações, retificações, estabilização de margens, dragagem e obras de proteção a enchentes), que cercearam parcialmente ou integralmente a dinâmica natural destes ecossistemas e que modificaram as suas condições de fluxo (BRAVARD et al, 2008, p. 954).

As políticas de gestão do território na Europa também não foram totalmente favoráveis à preservação dos cursos d'água, uma vez que elas permitiram, de certa forma, a modificação do uso do solo nas bacias hidrográficas, o aumento no tamanho das parcelas agrícolas, com a redução dos obstáculos ao escoamento superficial e o aumento da erosão dos solos, a drenagem dos fundos de vale e o desenvolvimento de áreas urbanas em áreas inundáveis (MINISTĖRE DE L'ÉCOLOGIE ET DU DÉVELOPPEMENT DURABLE, 2003, p. 2). Essa

\footnotetext{
${ }^{1}$ Geógrafo, doutor em Geografia pela Universidade de Lyon (França), professor na Universidade Federal de Pelotas.
} 
pressão sobre os recursos hídricos se intensificou principalmente após a Revolução Industrial (PIERRON e MONNIER, 2005, p. 3). Ao longo do século $\mathrm{XX}$, o crescimento populacional, a concentração da população nas cidades, a expansão das áreas agrícolas e a intensificação das atividades industriais acentuaram a transformação dos ambientes fluviais e fizeram com que governos e comunidades se deparassem com um aumento na demanda por recursos hídricos (GREGORY, 2008, p. 36). Frente a esse cenário, os pesquisadores e gestores de recursos hídricos dos países europeus, preocupados com a relação entre disponibilidade e demanda de recursos hídricos na Europa, concentraram esforços em busca de uma recuperação dos ambientes fluviais degradados, o que levou ao desenvolvimento dos primeiros trabalhos de restauração fluvial. Nas últimas décadas do século XX, a evolução dos conhecimentos sobre a dinâmica fluvial abriu caminho para o desenvolvimento de uma linha de pesquisas, políticas e práticas sobre essa temática. Recentemente, a publicação de algumas diretivas de gestão dos recursos hídricos por parte do Parlamento Europeu acelerou ainda mais este desenvolvimento.

O presente artigo é um breve apanhado das mais recentes discussões sobre restauração fluvial e gerenciamento dos recursos hídricos no âmbito dos países europeus, e tem por objetivo fazer uma reflexão sobre como países como o Brasil devem levar em consideração essas experiências para estabelecer os seus princípios de gestão dos recursos hídricos.

\section{Bases teóricas da restauração fluvial}

\subsection{O conceito de restauração fluvial}

O termo restauração fluvial vem sendo utilizado para identificar uma ampla gama de intervenções nos sistemas fluviais, desde uma simples intervenção local, como a construção de uma soleira, até intervenções em escalas mais amplas, como o desenvolvimento de um projeto integrado de recuperação de uma bacia hidrográfica. Muhar et al (1995, p. 184) definiram a restauração fluvial como sendo

a totalidade de medidas que mudam as alterações introduzidas pelo homem aos rios (primariamente as medidas de controle de cheias, mas também os desvios, os picos hidrológicos etc.) de maneira que o funcionamento ecológico do novo estado se pareça com um rio mais natural.

Para o European Centre for River Restoration (ECRR), 
a restauração fluvial se refere a uma ampla variedade de medidas que visam a restauração do estado natural, do funcionamento do rio e dos ambientes ripários. Restaurando as condições naturais, a restauração fluvial tem por objetivo estabelecer uma estrutura que permita o uso multifunctional sustentável dos rios (ECRR, 2008, p. 5).

Ainda existem muitas dúvidas sobre quais são os limites da restauração fluvial, tendo em vista que os objetivos de recuperação das condições ambientais podem variar muito de um rio para o outro, e variar também em diferentes escalas espaciais dentro de uma mesma bacia hidrográfica. Buscando tornar um pouco mais clara a definição de restauração fluvial, Brookes e Shields Jr (1996, p. 4) classificaram os objetivos de recuperação das condições ambientais em três conceitos diferentes:

- "Melhoria (Enhancement) - é qualquer melhoria na qualidade ambiental", como, por exemplo, uma melhora na qualidade dos parâmetros fisicoquímicos da água ou o reaparecimento de uma determinada espécie de peixes de interesse ecológico;

- "Reabilitação - é o retorno parcial para uma estrutura ou função prédistúrbio", como, por exemplo, a reconexão de um trecho de canal abandonado ao sistema fluvial;

- "Restauração - é o completo retorno a uma estrutura ou função prédistúrbio".

Em outra tentativa de síntese, Shields et al (2003) recolheram em diversos trabalhos a definição dos conceitos frequentemente utilizados na literatura sobre restauração fluvial (Tabela 1).

\section{Tabela 1 - Definição dos termos frequentemente associados com Restauração Fluvial}

\section{Termo} Definição

Restauração Restabelecimento da estrutura e da função dos ecossistemas. (Restoration) Restauração ecológica é o processo de retorno de um ecossistema o mais próximo possível das condições e das funções pré-distúrbio. Nos EUA, "pré-distúrbio" geralmente se refere ao período pré-colonização europeia. Considerando que os ecossistemas são dinâmicos, a perfeita replicação das condições prévias é impossível. 
Reabilitação Recuperação parcial das funções e processos do ecossistema. Projetos (Rehabilitation) de reabilitação incluem medidas estruturais e "recuperação assistida". Recuperação assistida se refere à remoção de uma perturbação básica ou de um distúrbio (por exemplo, a retirada de rebanhos de uma zona ripária), permitindo que os processos naturais (exemplo, crescimento da vegetação, processos fluviais) operem, levando a uma recuperação das funções do ecossistema.

Preservação Atividades para manter as funções correntes e as características de um (Preservation) ecossistema ou para protegê-lo de um futuro dano ou perda.

Mitigação Uma atividade para compensar ou amenizar um dano ambiental. A (Mitigation) mitigação pode ocorrer no local atingido pelo dano ou em qualquer outro lugar. Pode envolver a restauração do local para condições socialmente aceitáveis, mas não necessariamente para as condições naturais.

Naturalização Gestão que visa o estabelecimento de sistemas fluviais hidraulicamente (Naturalization) e morfologicamente variados, dinamicamente estáveis que sejam capazes de manter ecossistemas aquáticos biologicamente diversos. Não requer referência a um determinado estado preexistente.

Criação Formação de um novo sistema onde anteriormente não existia nenhum (Creation) (por exemplo, construção de uma área úmida).

Melhoria Termo subjetivo para as atividades empreendidas para melhorar a (Enhancement) qualidade ambiental existente.

Recuperação Uma série de atividades destinadas a modificar a capacidade biofísica (Reclamation) de um ecossistema. O ecossistema resultante é diferente do ecossistema que existia antes da recuperação.

Fonte: Shields et al (2003, p. 443)

Mesmo com esta tentativa de detalhamento, podemos dizer que ainda não existe um consenso entre os pesquisadores e gestores de recursos hídricos quanto ao conceito de restauração fluvial. Portanto, neste artigo, entenderemos a restauração fluvial como um mecanismo de gestão hidrográfica fundamentado nas teorias ecossistêmicas, onde o objetivo principal é a recuperação das funções ecológicas e hidro-geomorfológicas que controlam os ecossistemas fluviais, aproximando ao máximo o sistema fluvial dos padrões de qualidade ambiental observados em um estado de referência pré-definido. 


\subsection{Os princípios da elaboração de um projeto de restauração fluvial}

Existem inúmeras formas de se elaborar e conduzir um projeto de restauração fluvial. A definição do tipo de projeto a ser conduzido e das metodologias a serem empregadas vão depender diretamente dos objetivos de restauração definidos no início do trabalho e do nível de restauração que se pode e que se deseja atingir. Alguns projetos optam por uma restauração mais passiva, reduzindo as forças de degradação e deixando com que os rios se restituiam naturalmente. Há casos nos quais o uso da restauração passiva não é mais possível, pois o sistema fluvial já ultrapassou um limiar crítico de transformação, não conseguindo mais restabelecer as condições originais sem que haja uma força externa que o auxilie. Nestes casos, opta-se pelo desenvolvimento de projetos de restauração mais ativos, com a realização de intervenções diretamente no canal fluvial (ADAM et al, 2007a, p. 13).

Independente da abordagem adotada, é importante que alguns princípios básicos sejam observados ao longo de um projeto de restauração. Gregory (2008, pp. 36-37) estabeleceu seis princípios fundamentais para a elaboração e a execução de qualquer projeto de restauração fluvial:

- A restauração ecológica é projetada para um futuro mais ecológico e adequado à vida;

- A primeira prioridade do gerenciamento fluvial é a conservação da saúde dos componentes que mantêm o ecossistema funcionando;

- A restauração ecológica dos ecossistemas fluviais é baseada na restauração do dinamismo do rio, evitando práticas que dificultem ou limitem as trocas físicas e biológicas;

- A restauração fluvial deve incorporar práticas que sejam baseadas fundalmentalmente em questões fluviais e de rede, em vez de utilizar práticas baseadas no gerenciamento terrestre e de mosaicos paisagísticos;

- A restauração fluvial deve ser planejada, monitorada e adaptativamente gerenciada dentro de um escopo de múltiplas escalas espaciais de um rio que interage para criar um ecossistema fluvial;

- O gerenciamento fluvial deve antecipar as trajetórias futuras de mudanças e identificar também futuras trajetórias alternativas que sirvam de base para um gerenciamento adaptativo.

Para avaliar o sucesso de um projeto de restauração fluvial, Palmer et al (2005, p. 214) estabeleceram cinco princípios:

- O projeto deve estar baseado em uma imagem-guia de um rio mais saudável e dinâmico; 
- As condições ecológicas devem ser mensuravelmente melhoradas;

- O sistema fluvial deve ser mais auto-sustentável e resiliente a perturbações externas, exigindo mínima manutenção;

- Nenhum dano residual deve ser infligido ao ecossistema;

- As avaliações pré e pós-restauração devem ser concluídas e os dados devem ser tornados públicos.

\subsection{Métodos e técnicas de restauração fluvial}

Ao longo de um projeto de restauração fluvial, inúmeras técnicas podem ser utilizadas para promover uma melhoria da qualidade ambiental do sistema fluvial a ser restaurado. Considerando-se os princípios teóricos da restauração fluvial, que pregam uma adaptabilidade dos projetos às múltiplas escalas espaciais e contextos, a escolha das técnicas a serem utilizadas também vai depender dos objetivos da restauração e do nível de ambição desta restauração.

A partir de algumas experiências práticas de restauração fluvial, ADAM et al (2007b) elaboraram um conjunto de fichas técnicas sobre as principais intervenções utilizadas em projetos de restauração fluvial. Com base neste documento, é possível ter uma ideia da grande variedade de intervenções que podem ser realizadas em um projeto de restauração:

- Pequenas intervenções piscícolas: Trata-se da recriação de habitats favoráveis a reprodução e sobrevivência de espécies de peixes de especial interesse. Entre as intervenções que podem ser realizadas estão a criação de esconderijos e de áreas protegidas, com condições de fluxo favoráveis à espécie em questão;

- Criação da mata ripária: Considerada uma prática recomendável a qualquer curso d'água, a reconstituição da mata ripária pode ser feita de forma espontânea ou planejada, através do plantio de espécies de interesse ecológico e funcional.

- Uso de defletores de fluxo: Os defletores de fluxo podem ser criados com materiais naturais como pedras, galhos e mesmo com espécies vegetais adaptadas ao ambiente fluvial. Os defletores são utilizados para recentralizar o fluxo, para diversificar o substrato, para diversificar os níveis hidrométricos, para a criação áreas protegidas para a fauna piscícola, para guiar a dinâmica lateral do canal fluvial, entre outros objetivos; 
- Recriação das barras aluviais alternadas: A recriação das barras aluviais visa melhorar a diversidade de ambientes em canais retilíneos e muito homogêneos. As barras são criadas alternadamente, seguindo um espaçamento teórico próximo ao que seria o espaçamento natural entre barras em um canal natural de mesmas dimensões;

- Reconstituição da cobertura aluvial de fundo: A reconstituição do material aluvial de fundo é feita principalmente em canais nos quais a incisão do leito menor levou ao desaparecimento do material de fundo. Essa reconstituição pode ser feita através da promoção da erosão em zonas de estoque de sedimentos localizadas a montante ou pela reinjeção artificial de sedimentos no canal fluvial;

- Degraus e rampas: A construção de degraus e rampas de pedra no interior do canal é utilizada para evitar ou limitar a incisão do leito, principalmente nos rios de grande potência que foram submetidos a canalizações e ou dragagens excessivas. Os degraus e rampas também são importantes para a reconstituição do perfil longitudinal, para a uma diversificação do ambiente fluvial, para promover uma elevação do nível freático, entre outros objetivos;

- Reconexão dos anexos hidráulicos: Os anexos hidráulicos são zonas importantes do ponto de vista do funcionamento ecológico dos cursos d'água. A reconexão desses anexos é uma das operações mais complexas de um projeto de restauração e visa promover uma diversificação de habitats fluviais. A reconexão pode ser feita através da escavação dos obstáculos que impedem essa conexão, mas também pode ser feita através de intervenções que venham a corrigir os níveis hidrométricos do canal e do lençol freático;

- Redução/remoção de degraus: A redução ou remoção de degraus tem por objetivo reduzir o efeito barragem e restabelecer os fluxos hidrológico e biológico. A redução ou remoção de degraus também pode ser feita para corrigir o perfil longitudinal de um curso d'água;

- Supressão das perturbações laterais: Trata-se da remoção de quaisquer perturbações instaladas nas margens que impeçam o rio de exercer a sua dinâmica lateral e de ocupar o seu espaço de liberdade;

- Reabertura do canal a céu aberto: Trata-se da reabertura e reconstituição de um canal fluvial que foi canalizado ou coberto. A reabertura de um canal anteriormente coberto geralmente é uma intervenção difícil e requer a realização de trabalhos pesados de demolição e restauração;

- Modificação da geometria do leito menor/médio: Trata-se da readequação ou recriação da geometria do canal fluvial, em suas três dimensões, fazendo com que o canal restaurado se torne o mais próximo possível de um canal fluvial natural de mesmo porte; 
- Remoção de diques ou aumento do espaço entre diques: Trata-se da total remoção de diques e ou do aumento do espaço entre diques instalados ao longo dos canais fluviais. Tanto a remoção quanto o aumento do espaço entre os diques visa restaurar de forma total ou parcial a conexão do rio com o seu espaço de liberdade, restaurando também as funções ecológicas intrínsecas a esta dinâmica;

- Supressão de açudes: Os açudes representam um obstáculo no continuum fluvial, pois interferem na hidrometria, retêm sedimentos e nutrientes e podem dar fim à migração reprodutiva de algumas espécies. Assim sendo, a supressão de açudes visa a restabelecer a dinâmica natural montante-jusante de um sistema fluvial;

- Remeandramento ou recriação do curso d'água: Recomendada para os cursos d'água originalmente sinuosos e que perderam parcialmente ou totalmente a sua sinuosidade por conta das intervenções antrópicas. O remeandramento modifica consideravelmente a dinâmica hidráulica e sedimentar no interior do canal, resultando em uma maior variabilidade de ambientes, o que é desejável em um projeto de restauração fluvial.

\section{Europa e Brasil frente à restauração fluvial}

\subsection{A restauração fluvial na Europa: contextualização e estudos de caso}

A partir da década de 1980, os conhecimentos sobre geomorfologia, ecologia e hidrodinâmica fluvial apresentaram consideráveis avanços, o que de certa forma atraiu o interesse dos pesquisadores sobre os rios em estado natural. Esses novos conhecimentos também despertaram o interesse sobre a possibilidade de fazer com que rios já modificados pela ação humana voltassem a atingir o estado natural. Isto foi o ponto de partida para os projetos de restauração fluvial. Por conta do contexto da época, os primeiros projetos de restauração foram realizados sem muito conhecimento sobre a dinâmica dos sistemas fluviais, mas os trabalhos subsequentes passaram a ser realizados com um pouco mais de embasamento científico (BINDER, 2008, p. 97).

O interesse crescente dos países da União Europeia pela restauração fluvial é, em parte, resultado da orientação tomada pela política de meio-ambiente da Europa no decorrer das três últimas décadas e, em especial, nos últimos dez anos. De 1991 até a presente data, o Parlamento Europeu aprovou uma série de diretivas relacionadas ao uso, ao gerenciamento e à preservação dos recursos hídricos: 1991 - Nitratos; 1992 - Habitats; 2006 - Águas Balneáveis; 2006 - Águas Subterrâneas; 2007 - Cheias (GUMIERO et al, 2008, p. 3). Do ponto de vista da restauração fluvial, o momento mais importante foi a aprovação no parlamento da 
Diretiva Executiva Europeia sobre a Água, no ano de 2000 (PARLAMENTO EUROPEU, 2000). Essa diretiva previu a realização de um diagnóstico do estado atual dos sistemas fluviais europeus e a restauração dos sistemas que não forem considerados em bom estado até o ano de 2015. Face à dificuldade de cumprimento do objetivo proposto e ao horizonte de tempo relativamente curto, governos, instituições de pesquisas e pesquisadores imediatamente trouxeram a restauração fluvial para a pauta de discussões, de forma que atualmente existem inúmeros projetos de diagnóstico, classificação e restauração dos sistemas fluviais em andamento nos países da União Europeia.

Na França, a bacia do rio Rhône (Ródano) tem sido o objeto de uma série de estudos e projetos-piloto sobre dinâmica fluvial há mais de 25 anos (BRAVARD et al, 1999, p. 292; PIÉGAY et al, 2008, p. 68). Por conta das intervenções humanas, o canal do Rhône perdeu grande parte do seu espaço de liberdade ao longo dos últimos três séculos: no século XVIII, os diques de proteção de terras agrícolas cortaram a conexão das cheias com a planície fluvial; a partir da metade do século XIX, o canal sofreu inúmeras intervenções para o desenvolvimento da navegação (construção de diques laterais e submersos, fixação do canal, desconexão dos canais secundários, contrução de portos e atracadouros); a partir do final do século XIX e ao longo de todo o século XX, a bacia do Rhône recebeu uma série de barragens para a geração de energia elétrica (BRAVARD et al, 2008, p. 955). Nos anos 1970, o canal do Rhône foi intensamente explorado pela mineração de cascalhos, causando um déficit de sedimentos em alguns segmentos do canal fluvial (PIÉGAY et al, 2008, p. 69).

Desde a metade da década de 1990, uma série de projetos de restauração fluvial e de reconquista do espaço de liberdade do canal vem sendo conduzida na bacia do Rhône. Em 1997, a restauração dos ambientes fluviais alterados, principalmente das planícies fluviais, foi apontada como uma das dez prioridades de gestão no plano de gerenciamento da bacia do Rhône. Entre as mudaças no gerenciamento da bacia que foram adotadas visando à restauração do sistema fluvial, podemos citar a redução das obras de proteção de margens, permitindo uma retomada da erosão marginal e suprindo assim o déficit de sedimentos, a reintrodução artificial de sedimentos à jusante das barragens e nos canais desviados, o aumento das vazões mínimas defluentes e a elevação do leito fluvial a fim de permitir a reativação dos meandros abandonados, entre outras iniciativas. Atualmente, uma proposta de alargamento de alguns trechos do canal do Rhône encontra-se em discussão (BRAVARD et al, 2008). As experiências de gestão e restauração realizadas na bacia do Rhône e em outras bacias hidrográficas da França resultaram em uma série de documentos e manuais técnicos muito úteis para aqueles que necessitam de um ponto de partida para a elaboração e a 
execução de projetos de restauração fluvial (BOYER et al, 1998; MALAVOI et al, 1998; PIERRON e MONNIER, 2005; ADAM et al, 2007 a e 2007b).

Na Dinamarca, o rio Skjern, o maior do país, foi objeto de um importante projeto de restauração fluvial. Nos anos 1960, o baixo vale do rio Skjern, originalmente uma área de relevo suave recoberto por campos e vegetação de áreas alagadas, com canais meandrantes e lagoas rasas, foi transformado em área agrícola pelo maior projeto de drenagem da Dinamarca. Por conta disto, $40 \mathrm{~km}^{2}$ de áreas úmidas foram drenadas por bombeamento, o canal do rio Skjern foi retificado, aprofundado e canalizado, desconectando o rio de sua planície fluvial (PEDERSEN et al, 2007, pp. 131-132; ECRR, 2008, pp. 16-17).

Em 1987, o Parlamento dinamarquês decidiu pela restauração do baixo rio Skjern e do seu vale. Os objetivos gerais a serem atingidos por essa restauração eram os seguintes: 1) restaurar a capacidade de retenção de nutrientes do rio e do seu vale; 2) restaurar uma área úmida de importância internacional como habitat de aves migratórias; 3) promover o aumento da população de peixes no estuário Ringkфbing Fjord, localizado à jusante e; 4) aumentar o valor turístico e recreacional na área do projeto. Em 1997, uma proposa de projeto de restauração foi elaborada pela agência florestal e, em 1998, um estudo de impactos ambientais foi elaborado por essa mesma agência. Esses documentos, somados às ideias e propostas recolhidas nas audiências públicas, foram incorporados ao projeto original do Parlamento dinamarquês. Os trabalhos de restauração começaram efetivamente em junho de 1999 e se extenderam até o último trimestre de 2002 (PEDERSEN et al, 2007, pp. 131-132).

Dentre as principais atividades de restauração realizadas no rio Skjern, podemos citar a construção de $40 \mathrm{~km}$ de um novo canal fluvial em padrão meandrante, em alguns trechos seguindo o mais próximo possível o traçado de antigos meandros mapeados no século XIX, a reconexão do canal principal com a planície fluvial através da remoção de diques e das casas de bombeamento, a criação de ambientes lacustres rasos nas áeras adjacentes ao canal, a restauração da vegetação de campos e de áreas úmidas, entre outras atividades (ECRR, 2008, p. 18). Após a execução dos trabalhos de restauração, foram estabelecidos um plano de manejo para a área restaurada e um amplo programa de monitoramento, a fim de identificar as mudanças ambientais consequentes do processo de restauração. As conclusões de curto prazo desse monitoramento mostraram que os trabalhos de restauração provocaram relativas melhorias na qualidade ambiental geral do rio Skjern, mas que algumas variáveis foram afetadas negativamente pela restauração. Quanto aos impactos econômicos, as estimativas mostram que o custo da restauração (38 milhões de euros) será amortizado ao longo dos próximos 20 anos. Atualmente, o número de visitantes na área do projeto chega a 100 mil pessoas por ano, e espera-se que esse valor aumente nos próximos anos, o 
que ajudará a recuperar os custos da restauração em um período mais curto (PEDERSEN et al, 2007, p. 141; ECRR, 2008, p. 19).

Além do Rhône e do Skjern, outros importantes rios europeus vêm sendo submetidos a projetos de restauração fluvial, como é o caso do Reno, do Danúbio e do Arno. Os objetivos de restauração e as metodologias e técnicas empregadas na restauração desses rios variam de caso para caso, mas é possível descrever que todos esses projetos têm em comum a busca por uma recuperação da qualidade ambiental dos sistemas fluvias e um esforço de reconciliação dos países da União Europeia com os seus cursos d'água.

\subsection{O Brasil e a restauração fluvial: o começo de um debate}

A restauração fluvial é um tema praticamente ausente das discussões sobre a gestão dos recursos hídricos no Brasil. Isso se justifica até certo ponto, uma vez que, em relação ao continente europeu, o Brasil é considerado um país "novo", em pleno desenvolvimento, de forma que as nossas prioridades políticas, econômicas e de gestão dos nossos recursos hídricos não são exatamente as mesmas dos países da União Europeia. Mesmo assim, o Brasil deu nos últimos 20 anos alguns passos importantes para a reestruturação do sistema de gestão dos recursos hídricos, com a elaboração da Política Nacional de Recursos Hídricos, a criação de toda a base institucional responsável pela colocação em prática dessa política e a publicação da primeira versão do Plano Nacional de Recursos Hídricos. Considerando-se esses pressupostos, nos colocamos então o seguinte questionamento: Por que o Brasil deve se interar das discussões sobre restauração fluvial?

Por ser um país em desenvolvimento, as políticas de gestão dos recursos hídricos no Brasil priorizam as questões de cunho estratégico, como, por exemplo, a relação entre demanda e disponibilidade de água ou a qualidade de água para o abastecimento humano, a geração de energia, deixando em segundo plano as questões referentes à qualidade ambiental global dos sistemas fluviais e o papel ecológico desempenhado por estes.

Segundo Gregory (2008, p. 44), “A restauração fluvial vai desempenhar um papel cada vez mais importante no gerenciamento fluvial à medida que os rios do mundo estiverem mais e mais degradados". Se pensarmos no caso do Brasil, ainda estamos em uma situação confortável, mas a pressão sobre os recursos hídricos vem aumentando progressivamente (ANA, 2009, pp. 186-187). Por conta disso estamos nos distanciando cada vez mais de nossos sistemas fluviais: nossas intervenções ainda são projetadas para reduzir a dinâmica natural dos rios, mantendo-os imóveis lateralmente e verticalmente e reduzindo a sua variabilidade hidrológica. Acompanhar as discussões sobre restauração fluvial que acontecem 
na Europa e em alguns outros países ao redor do mundo pode ser um ponto de partida para que o Brasil reveja algumas de suas práticas de gestão dos recursos hídricos, além de ser prudente do ponto de vista preventivo, pois não devemos esperar que os nossos sistemas fluviais sejam completamente alterados para posteriormente pensarmos em restauração de suas funções físicas e ecológicas. No presente momento, o mais próximo que o Brasil tem chegado da restauração fluvial são alguns programas de despoluição de algumas bacias hidrográficas, como por exemplo, no Rio das Velhas e no rio Tietê. Apesar de já terem apresentados alguns avanços, esses programas ainda irão requerer mais esforços nos próximos anos, principalmente financeiros (ANA, 2009, p. 187).

É hora de o Brasil se inspirar no exemplo europeu e buscar também uma reconciliação com os seus sistemas fluviais, gerenciando-os de uma forma mais holística e evitando, assim, a necessidade de restauração num futuro próximo.

\section{Conclusões}

"A restauração fluvial é uma ciência relativamente nova e, por isto, recém está começando a ser reconhecida cientificamente e profissionalmente" (DOYLE et al, 1999, p. 4). A evolução dos conhecimentos sobre a dinâmica fluvial e a publicação da Diretiva Executiva Europeia sobre a Água deram um grande impulso a essa ciência, principalmente na Europa, onde a necessidade de restauração a colocou no topo da pauta de discussão sobre gestão de recursos hídricos. Atualmente, os países da União Europeia têm dispensado parcelas importantes dos seus recursos em projetos de restauração fluvial, na busca do cumprimento das metas estabelecidas pela Diretiva Executiva Europeia sobre a Água.

Com o avanço das discussões, naturalmente já surgem algumas críticas à restauração fluvial, e alguns autores têm questionado principalmente a forma como alguns projetos de restauração vêm sendo conduzidos, privilegiando o caráter estético e negligenciando as funções físicas e ecológicas dos sistemas fluviais (DOYLE et al, 1999; LACHAT, 2008; PALMER, 2008). Apesar das críticas e de alguns projetos mal executados, a restauração fluvial vem se mostrando uma ciência cada vez mais importante na gestão dos sistemas fluviais e, quando bem conduzida, pode promover visíveis melhorias na qualidade ambiental dos ecossistemas em questão. As críticas têm permitido ainda que as pesquisas e políticas caminhem em direção a um ponto em comum: os projetos de restauração devem ser planejados e implantados de forma a manter e restaurar os processos físicos e biológicos que criam ecossistemas saudáveis e habitats com alta qualidade ambiental (ALLAN e CASTILLO, 2007, p. 350). Com a chegada 
dos primeiros resultados dos projetos de restauração mais antigos, os métodos e técnicas de restauração vêm sendo reavaliados, de forma a garantir que os novos projetos obtenham maior êxito na busca pela melhoria da qualidade ambiental global dos sistemas fluviais.

No Brasil, um país considerado "novo", a restauração fluvial ainda é praticamente desconhecida. Entretanto, isso não exclui a possibilidade de termos de recorrer a essa ciência em um futuro próximo, pois a pressão sobre os recursos hídricos aumenta a cada ano que passa, consequentemente levando a um progressivo aumento da degradação de nossos sistemas fluviais. Diferentemente do Brasil, alguns países também considerados "novos" já se adiantaram e estão se envolvendo desde já com as pesquisas sobre restauração fluvial, como é o caso do México e da Austrália. Seria interessante que os pesquisadores e responsáveis pela gestão dos recursos hídricos no Brasil também passassem a acompanhar e a participar de forma mais próxima dessas discussões, a fim de aprender com os erros e acertos das experiências europeias e para, em breve, adotar novas formas de gestão dos recursos hídricos, antecipando o país para os possíveis desafios futuros.

\section{Agradecimentos}

O autor deste artigo agradece aos colegas Débora Pinto Martins, Sínthia Cristina Batista e Eduardo Pinheiro da Silva pelas revisões e sugestões apresentadas. O autor ainda agradece ao professor Jean-Paul Bravard, pela formação recebida sobre a temática deste artigo, pelas sugestões e pelo envio de materiais. Agradeço também à unidade mista de pesquisa UMR 5600 Environnement, Ville, Société, da Universidade de Lyon, que me recebeu para a realização do doutorado e onde recebi parte da minha formação em dinâmica fluvial.

\section{A RESTAURAÇÃO FLUVIAL E A BUSCA DE RECONCILIAÇÃO DA EUROPA COM OS SEUS CURSOS D'ÁGUA: O QUE O BRASIL DEVE APRENDER COM ESTA EXPERIÊNCIA?}

Resumo: A restauração fluvial é um assunto relativamente desconhecido no Brasil, mas nos países da União Europeia e em alguns países desenvolvidos ele vem sendo um dos temas prioritários na pauta de discussões das autoridades responsáveis pela gestão dos recursos hídricos. O presente artigo é um breve apanhado das mais recentes discussões sobre restauração fluvial e o gerenciamento dos recursos hídricos no âmbito dos 
países europeus, e tem por objetivo fazer uma reflexão sobre como países como o Brasil devem levar em consideração essas experiências para estabelecer os seus princípios de gestão dos recursos hídricos.

Palavras-chave: restauração fluvial, gestão de recursos hídricos, dinâmica fluvial, ecologia fluvial.

"THE FLUVIAL RESTORATION AND THE SEARCH OF EUROPE TO RECONCILIATE WITH THEIR RIVERS: WHAT BRAZIL SHOULD LEARN FROM THIS EXPERIENCE ?"

Abstract: Fluvial restoration is a relatively unknown subject in Brazil, but in the countries of the European Union and in some other developed countries the subject is one of the priority issues in the discussions of the authorities responsible for the water resources management. The following paper is a brief synthesis of the most recent discussions about fluvial restoration and about the water resources management in the European countries, and it aims to reflect how countries like Brazil should learn from these experiences and establish their own principles of water resources management.

Keywords: fluvial restoration, management of the water resources, fluvial dynamics, fluvial ecology

\section{BIBLIOGRAFIA}

ALlAN, J. D. e CASTILlO, M. M. (2007) Stream Ecology: Structure and function of running waters. Springer. Dordrecht.

ADAM, P.; DEBIAIS, N.; MALAVOI, J.-R. (2007a) Manuel de Restauration Hydromorphologique des Cours d'Eau. Agence de l'Eau Seine-Normandie. Nanterre.

.(2007b) Manuel de Restauration Hydromorphologique des Cours d'Eau: Guide de Terrain. Agence de l'Eau Seine-Normandie. Nanterre.

ANA - AGÊNCIA NACIONAL DE ÁGUAS. (2009) Conjuntura dos recursos hídricos no Brasil 2009. Brasília.

BINDER, W. (2008) River Restoration: an European overview on rivers in urban areas. In.: GUMIERO, B.; RINALDI, M.; FOKKENS, B. Proceedings of $4^{\text {th }}$ ECRR Conference on River Restoration 2008. ECRR - European Centre for River Restoration / CIRF - Centro Italiano per la Riqualificazione Fluviale. Veneza.

BOYER, M.; PIEGAY, H.; RUFFINONI, C. CITTERIO, A.; BOURGERY, C.; CAILlEBOTE, P. (1998) La gestion des boisements de rivière: dynamique et 
fonctions de la ripisylve. Guide technique SDAGE $n^{\circ} 1$. Agence de l'Eau RhôneMéditerranée-Corse. Lyon.

BRAVARD, J.-P.; COLLILIEUX, G.; DESMET, M.; FRUCHART, F.; MOTCHALOVA, O; VALÉ, N. (2008). Widening By-passed Reaches of the Rhone River, France, Removing Sediment Deposits. Interdisciplinary studies and procedures. In.: GUMIERO, B.; RINALDI, M.; FOKKENS, B. Proceedings of $4^{\text {th }}$ ECRR Conference on River Restoration 2008. ECRR - European Centre for River Restoration / CIRF - Centro Italiano per la Riqualificazione Fluviale, pp. 953963. Veneza.

BRAVARD, J.-P.; LANDON, N.; PEIRY, J. -L.; PIÉGAY, H. (1999) Principles of engineering geomorphology for managing channel erosion and bedload transport, examples from French rivers. Geomorphology, 31, pp. 291-311.

BROOKES, A. e SHIELDS JR, F.D. (eds.) (1996) River Channel Restoration: Guiding principles for sustainable projects. Wiley. Chichester.

DOYLE, M. W.; MILLER, D. E.; HARBOR, J. M. (1999) Should River Restoration Be Based on Classification Schemes or Process Models? Insights from the history of geomorphology. ASCE International Conference on Water Resources Engineering. ASCE, 1999, pp.1-9. Seattle.

EUROPEAN CENTRE FOR RIVER RESTORATION. (2008) ECRR Addressing Practitioners. ECRR - European Centre for River Restoration. Lelystad.

GREGORY, S.(2008), River Restoration: restoring dynamic riverine processes in a changing world... or erecting monuments to our good intentions. In: GUMIERO, B.; RINALDI, M.; FOKKENS, B. Proceedings of $4^{\text {th }}$ ECRR Conference on River Restoration 2008, ECRR - European Centre for River Restoration / CIRF - Centro Italiano per la Riqualificazione Fluviale, pp. 35-46. Veneza.

GUMIERO, B.; RINALDI, M.; FOKKENS, B. (2008) Proceedings of $4^{\text {th }}$ ECRR Conference on River Restoration 2008. ECRR - European Centre for River Restoration / CIRF - Centro Italiano per la Riqualificazione Fluviale. Veneza.

LACHAT, B. (2008) Revitalisation des cours d'eau: A l'exemple de la Birse et ses affluents. GWA, 3, pp. 199-205.

MALAVOI, J. R.; BRAVARD, J. -P.; PIÉGAY, H.; HÉROUIN, E.; RAMEZ, P. (1998) Détermination de l'espace de liberté des cours d'eau. Guide technique $S D A G E \mathrm{n}^{\circ} 2$, Agence de l'Eau Rhône-Méditerranée-Corse, 39 p. Lyon.

MINISTÈRE DE L'ÉCOLOGIE ET DU DÉVELOPPEMENT DURABLE. (2003) La restauration et l'entretien des cours d'eau: Une gestion écologique. Ministère de l'Écologie et du Développement Durable / Direction de l'Eau. Paris.

MUHAR, S.; SCHMUTZ, S.; JUNGWIRTH, M. (1995) River restoration concepts - goals and perspectives. Hydrobiologia, 303, pp. 183 - 194. 
PALMER, M. A.; BERNHARDT, E. S.; ALLAN, J. D.; LAKE, P. S.; ALEXANDER, G.; BROOKS, S.; CARR, J.; CLAYTON, S.; DAHM, C. N.; FOLLSTAD SHAH, J.; GALAT, D. L.; LOSS, S. G.; GOODWIN, P.; HART, D. D.; HASSETT, B.; JENKINSON, R.; KONDOLF, G. M.; LAVE, R.; MEYER, J. L.; O'DONNELL, T. K.; PAGANO, L.; SUDDUTH, E. (2005) Standards for ecologically successful river restoration. Journal of Applied Ecology, 42, pp. 208217.

PALMER, M. A. River Restoration as a Collaboration with Nature. In: GUMIERO, B.; RINALDI, M.; FOKKENS, B.(2008) Proceedings of $4^{\text {th }}$ ECRR Conference on River Restoration 2008. ECRR - European Centre for River Restoration / CIRF - Centro Italiano per la Riqualificazione Fluviale, pp. 27-33. Veneza.

PARLAMENTO EUROPEU. (2000) Directive 2000/60/EC of the European Parliament and of the Council establishing a framework for Community action in the field of water policy. O.J.L, 327.

PEDERSEN, M. L.; ANDERSEN, J. M.; NIELSEN, K.; LINNEMANN, M. (2007) Restoration of Skjern River and its valley: Project description and general ecological changes in the project area. Ecological Engineering, 30, pp. 131-144.

PIÉGAY, H.; BORNETTE, G.; LAMOUROUX, N.(2008) Geomorphology and River Restoration Feedback and Outstanding Issue in the Hydrographic District of the Rhone River. Proceedings of $4^{\text {th }}$ ECRR Conference on River Restoration 2008. ECRR - European Centre for River Restoration / CIRF - Centro Italiano per la Riqualificazione Fluviale, pp.67 - 82. Veneza.

PIERRON, F. e MONNIER, D. (2005) Restauration Physique des Cours d'Eau dans le Nord-Est de la France. Conseil Supérieur de la Pêche - Délégation Régionale de Metz. Marly.

SHIELDS, F. D.; COOPER Jr., C. M.; KNIGHT, S. S.; MOORE, M. T.(2003) Stream Corridor Restoration Research: a long and winding road. Ecological Engineering, 20, pp. 441-454. 